\title{
Germanica
}

\section{Les avatars des projets de langue universelle au tournant du siècle (1880-1930)}

Höhe- und Tiefpunkt der Weltsprachenprojekte um die Jahrhundertwende (1880-1930)

\section{André Rousseau}

\section{OpenEdition}

\section{Journals}

Édition électronique

URL : http://journals.openedition.org/germanica/1840

DOI : 10.4000/germanica. 1840

ISSN : 2107-0784

\section{Éditeur}

Université de Lille

\section{Édition imprimée}

Date de publication : 31 décembre 2003

Pagination : 213-230

ISBN : 9782913857124

ISSN : 0984-2632

Référence électronique

André Rousseau, "Les avatars des projets de langue universelle au tournant du siècle (1880-1930) », Germanica [En ligne], 33 | 2003, mis en ligne le 04 décembre 2012, consulté le 06 octobre 2020. URL: http://journals.openedition.org/germanica/1840; DOI : https://doi.org/10.4000/germanica.1840

Ce document a été généré automatiquement le 6 octobre 2020.

(c) Tous droits réservés 


\section{Les avatars des projets de langue universelle au tournant du siècle (1880-1930)}

Höhe- und Tiefpunkt der Weltsprachenprojekte um die Jahrhundertwende

(1880-1930)

André Rousseau

1 La langue universelle, vieux rêve de l'humanité depuis Babel, apparaît bien avant le XIX siècle, principalement avec Raymond Lulle (1235-1315) et G.W. Leibniz (1646-1716) ${ }^{1}$, pour ne citer que deux figures de proue ; mais les essais en tout genre culminent entre 1880 et 1930. Nous situerons cette question au confluent de deux axes de réflexion qui s'inscrivent l'un et l'autre dans le temps, qui apparaît comme la référence naturelle dans laquelle prennent corps et s'organisent toute pensée et tout acte de langage :

- l'un tourné vers le passé met directement en jeu la mémoire, cultivée et pratiquée depuis la plus haute Antiquité comme ars memorativa, dont on attribue la paternité à Simonide de Céos (556-467). La mémoire, dont Piaget a su montrer le lien avec les principales fonctions cognitives sous forme de schèmes, a pour rôle essentiel d'évaluer le temps tout en l'effacant ;

- l'autre considérant au contraire le temps comme animé d'une dynamique irréversible, représentant une marche en avant, synonyme de progrès, atteint son apogée au XVIII ${ }^{\mathrm{e}}$ siècle et anime l'Encyclopédie et tous les Encyclopédistes.

2 À vrai dire, les langues, qu'elles soient naturelles ou artificielles, n'échappent pas à cette double problématique qui devrait être au cœur de la philosophie du langage, mais qui reste la plupart du temps éludée, comme si les spécialistes reculaient devant la difficulté inhérente à cette vaste question : Que signifie en profondeur l'inscription des langues dans le temps?

3 Avant de pouvoir tenter d'apporter un début de réponse à cette interrogation, même limitée aux langues artificielles $^{2}$, il est nécessaire de rappeler les conditions d'apparition des langues artificielles et d'en proposer une typologie : langue logique, langue mixte ${ }^{3}$, langue artificielle à base naturelle. La conclusion reste prudente et 
mesurée, car le temps n'est pas automatiquement pour les langues vecteur de progrès, surtout si les langues offrent une évolution circulaire - comme nous l'avons suggéré par ailleurs (1999b).

\section{Les données initiales de la question}

4 Si l'on s'interroge pour déterminer à quelle(s) nécessité(s) fait écho la naissance des langues artificielles, il semble que les motifs peuvent être de plusieurs ordres :

- soit il peut s'agir d'un idéal universaliste à étendre à l'humanité, comme cela a pu être le cas dans l'enthousiasme de la Révolution, lorsque Jean Delormel présente son Projet d'une langue universelle à la Convention en 1795) ;

- soit cet idéal peut être quasiment mystique, puiser aux sources de la foi, en voulant enfin libérer les hommes et effacer le châtiment de Babel ;

- soit les causes peuvent être intellectuelles, culturelles ou scientifiques et la langue universelle est alors destinée à prendre le relais du latin, qui était la langue internationale de la communauté scientifique au Moyen Âge comme à la Renaissance et même largement au-delà ${ }^{\text {; }}$

- soit enfin il peut y avoir des raisons économiques ou politiques, voire militaires, qui se présentent souvent au cours de l'histoire, même à l'époque actuelle; pour prendre un exemple ancien qui ne choquera plus les susceptibilités, les Romains ont imposé le latin ou tenté de le faire dans tous les pays où se sont exercées leurs conquêtes.

\subsection{Forces et faiblesses des langues naturelles}

Les langues naturelles sont des objets remarquables par leur adaptation à toutes les situations, créés par la faculté de langage inhérente à tout être humain, qui contiennent et expriment en même temps la mémoire d'un groupe donné, d'une certaine communauté linguistique. Ce trait, qui a été souligné tout au long de l'histoire, est d'ailleurs facile de démontrer: le lexique d'une langue emmagasine les grandes expériences du groupe qui en est le support; ainsi en allemand, quelques mots bien choisis comme essen, sitzen, ou Eisen, Erbe, ou encore Strasse, Ziegel ou bien zwölf, tausend, ou même Schule, schreiben, etc. révèlent par eux-mêmes les grands moments de l'histoire des tribus germaniques et des populations allemandes.

Le second volet est beaucoup plus délicat et il est prudent de le formuler sous forme de question: l'évolution des langues naturelles peut-elle être considérée comme un progrès? Certains penseurs n'hésitent à placer les langues sous le signe du progrès ; cette idée réapparait chez divers linguistes appartenant à différentes époques et à différents domaines, qu'il s'agisse de Leibniz (1646-1716), qui parle de « Verbesserung der deutschen Sprache ", ou de Léon Clédat (1851-1930), qui discerne un progrès dans l'évolution de la langue, sans que celui-ci soit pour autant linéaire et continu, ou également d'Aleksander Potebnja (1835-1891), qui conçoit le progrès comme cumulatif, ou encore d'Otto Jespersen (1860-1943), qui publie Progress in Language (1894) où il défend la thèse $\mathrm{du}$ progrès historique des langues allant dans le sens de la simplification. Cette conception, qui se manifeste principalement dans la seconde moitié $\mathrm{du} \mathrm{XIX}^{\mathrm{e}}$ siècle, est une réaction contre la vision romantique des premiers comparatistes allemands au début du siècle qui concevaient l'évolution des langues comme une dégradation constante des trésors transmis originellement à l'humanité. 


\subsection{Les défauts des langues naturelles}

$7 \quad$ Pour établir une langue universelle parfaite ${ }^{5}$, deux types de courants adressent chacun leurs critiques spécifiques aux langues naturelles. La mise en cause de celles-ci est en effet menée sur deux fronts : au nom de la logique d'abord et au nom d'une langue universelle.

\subsubsection{Critiques émanant de la logique}

Les logiciens sont unanimes à reprocher aux langues naturelles leur manque de rigueur et de précision. Ce n'est que par rapport à l'entendement que les langues sont dites 'imparfaites', comme si les mots devaient être les signes des idées et ce n'est que sous ce rapport qu'on peut envisager de construire une langue parfaite. À cet égard, toutes les langues naturelles sont placées au même niveau.

\subsubsection{Critiques issues des tenants d'une langue internationale}

Le constat de l'inadéquation des langues naturelles s'explique surtout par leur grande dispersion; le reproche tient ici en un mot: les langues naturelles, bien que leurs divergences soient de nature variable, sont 'babélisées' et représentent un obstacle à la libre communication entre les hommes, au 'libre-échangisme' linguistique.

\subsection{De quelques projets antérieurs}

Dans cette vaste ambition de langue universelle, aux multiples ramifications, il faut naturellement évoquer la 'grammaire générale', dont le dessein est précisément d'embrasser « les raisons de ce qui est commun à toutes les langues et des principales différences qui s'y rencontrent ». Il serait légitime également de parler des 'langues imaginaires'.

11 Deux Allemands méritent une attention particulière pour leurs projets de langue universelle : l'un est philosophe et son nom a déjà été évoqué, il s'agit de G.W. Leibniz ; l'autre est un linguiste très connu, mais peu de gens savent qu'il s'est aussi intéressé à la langue universelle, il s'agit de Jacob Grimm (1785-1863).

\subsubsection{Leibniz et la Lingua characteristica universalis}

12 Les recherches de Leibniz sur la langue universelle s'appuient sur les grammaires des différentes langues vernaculaires, notamment le latin, qui seront soumises à une simplification. L'essentiel des travaux de Leibniz réside dans le rapport qu'il établit entre grammaire et logique et qu'il examine à deux niveaux qui se correspondent: d'une part, il procède à une analyse des flexions et des particules et à une analyse parallèle des relations logiques; d'autre part, il propose « une analyse des concepts au moyen de la définition des mots». Dans un dialogue entre Théophile et Philalèthe, Leibniz fait dire à Théophile: "Mais pour revenir à vos quatre défauts de la nomination, je vous dirai, Monsieur, qu'on peut remédier à tous, surtout depuis que l'écriture est inventée et qu'ils ne subsistent que par notre négligence. Car il dépend de nous de fixer les significations, au moins dans quelque langue savante, et d'en convenir 
pour détruire cette tour de Babel.» (Nouveaux Essais sur l'entendement humain. Livre III ; chap. IX)

Certes, le second niveau, comme le rappelle Fr. Nef, est également présent chez Descartes : «L'invention de cette langue [= universelle] dépend de la vraie Philosophie ; car il est impossible autrement de dénombrer toutes les pensées des hommes, ni seulement de les distinguer en sorte qu'elles soient claires et distinctes. " (Lettre à Mersenne du 20 novembre 1629).

L'objectif final de cette double analyse chez Leibniz est d'arriver à proposer une Langue Universelle, c'est-à-dire un langage qui ne trahisse pas la pensée: c'est en fait l'aspiration secrète de tous les penseurs depuis Aristote. Mais sa réussite est naturellement subordonnée à la mise sur pied d'une grammaire rationnelle.

Même si la réalisation de cet immense projet n'a abouti qu'à des échantillons partiels, loin de la perfection recherchée, il faut souligner l'innovation que représente cette vaste entreprise, qui marque une date capitale dans l'histoire de la logique.

\subsubsection{Jacob Grimm et son Programme pour la formation d'une langue universelle}

Jacob Grimm est réputé pour la publication de sa Deutsche Grammatik en quatre volumes (1819-1838), qui a fait de lui le fondateur de la grammaire comparée des langues germaniques, et aussi comme co-auteur avec son frère Wilhelm du Deutsches Wörterbuch, achevé seulement en 1963. Il est beaucoup moins connu pour avoir dressé, peu de temps avant sa mort, un 'Programme' destiné à créer une langue universelle (1860).

17 Les raisons qui conduisent Jacob Grimm à proposer ce programme sont au nombre de trois :

- «les avantages extraordinaires qui résulteraient pour tout le genre humain de la formation et de l'adoption d'une langue universelle »

- «aucune des langues connues jusqu'ici ne peut être employée à cette fin »

- «tous les essais faits jusqu'ici dans ce sens ont nécessairement échoué »

18 Tout à fait conscient des difficultés de l'entreprise, Grimm expose néanmoins les propriétés qu'il reconnaît à cette langue :

1. Elle doit être rigoureusement logique dans le rapport du mot au concept et dans la formation des mots (composition et dérivation);

2. Elle doit être d'une richesse illimitée dans le vocabulaire et dans l'ordre des mots.

3. Elle doit être harmonieuse et appropriée à la poésie et au chant.

4. Elle doit être extrêmement facile à apprendre, à parler et à écrire.

19 Pour tenir compte de ces conditions, le latin lui parait être la langue la plus propre à servir de fil conducteur pour la formations des racines essentielles.

20 Mais en bon linguiste, Grimm se pose quelques questions délicates: «le nom doit-il toujours être formé à partir du verbe, ou inversement ? [...] La racine doit-elle être toujours formée du concept primitif, ou peut-être du concept abstrait ?».

Le programme de Grimm apparaît frappé au coin du bon sens, mais la réalisation est beaucoup plus difficile que ne l'imaginait Grimm lui-même. Certains promoteurs des langues universelles ont tenté de réaliser une langue proche des principes de Grimm : ainsi, G. Peano et son latin simplifié (De latino sine flexione, 1903). 


\subsection{La situation linguistique au tournant du siècle} axes très différents, apparemment sans recoupement possible.

\subsubsection{La grammaire historique}

Des travaux de grande ampleur sont entrepris par les néo-grammairiens, surtout allemands, sur les périodes anciennes de toutes les langues indo-européennes, dans les principaux domaines :

-en phonétique historique, où domine la toute-puissance des 'lois phonétiques', comme l'expriment Osthoff et Brugmann, à la suite de Leskien qui avait proclamé en premier le caractère sans exception des 'lois phonétiques' (1876),: «Aller lautwandel, so weit er mechanisch vor sich geht, vollzieht sich nach ausnahmslosen gesetzen [...]. » (1878, Vorwort)

- en morphologique historique, comme en témoigne l'ouvrage en cinq volumes des mêmes Osthoff et Brugmann: Morphologische Untersuchungen auf dem Gebiete der indogermanischen Sprachen (1878-1890)

- en syntaxe historique, avec les études de B. Delbrück sur la syntaxe des langues i. -e. et celles d'O. Behaghel (Deutsche Syntax 4 vol. 1923-1932) ;

-en étymologie avec l'apparition des premiers dictionnaires étymologiques, comme par exemple celui de Friedrich Kluge (1883).

Après 1925, l'orientation linguistique représentée par les néo-grammairiens est sur le déclin, car les idées de Saussure (1857-1913), diffusées par la publication du Cours de linguistique générale $(1916)^{7}$, commencent à faire leur chemin.

\subsubsection{Les langues artificielles}

Pendant cette période se manifeste une activité sans précédent, mobilisant logiciens, linguistes et autres ${ }^{8}$, pour l'établissement de langues artificielles, qu'il est quasiment impossible de recenser exhaustivement, tant leur nombre est élevé, aussi bien en France qu'en Allemagne ${ }^{9}$. Il y a eu une "véritable Babel des langues artificielles", comme le note U. Eco.

Ainsi on peut citer, à titre d'exemples, quelques essais émanant soit de logiciens connus : l'Ido de Beaufront et Couturat (1908), l'Interlingua de Peano (1909), soit de linguistes de renom : l'Apoléma de Raoul de la Grasserie ${ }^{10}$ (1907), le Novial de Jespersen (1928).

Le point de départ officiel de ces tentatives se situe à Paris, lors du Congrès international de philosophie en 1900, à l'initiative de Louis Couturat, qui avait invité Frege - qui ne viendra pas - et qui fera créer la 'Délégation pour l'adoption d'une langue auxiliaire internationale', organisme qui exercera un rôle moteur pour le développement d'une langue internationale jusqu'en 1915, année du décès de Couturat.

La question essentielle qui se pose pour l'élaboration d'une langue internationale est de savoir sur quelle langue européenne on peut s'appuyer : les langues slaves sont peu répandues et assez difficiles à apprendre; des langues germaniques, seul l'anglais offre un grand intérêt en raison de son absence relative de morphologie; les langues 
romanes sont diverses, mais on connait leur origine commune, le latin, ce qui est un cas assez exceptionnel.

On comprend dès lors que de nombreuses langues internationales de l'époque aient opté pour une base latine.

\section{Les langues universelles en logique : Frege}

Ces langues sont 'universelles' dans la mesure où elles sont le véhicule de la 'pensée pure' et elles s'opposent, selon Frege ${ }^{11}$, aux langues naturelles «qui n'offrent aucune aide certaines pour les questions logiques. Et ce n'est pas une des moindres tâches du logicien que de montrer quelles embûches le langage a préparées à la pensée. » ( $L a$ négation, 1918)

\subsection{La nouveauté de la Begriffsschrift (1879) de Frege}

31 C'est Gottlob Frege (1848-1925), renouant avec la tradition leibnizienne, qui propose en 1879 - date fatidique qui coïncide avec la naissance du Volapük du prélat allemand Martin Schleyer -, et alors qu'il n'était que Privatdozent de mathématiques à Iéna, une Begriffsschrift ('idéographie'), qu'il définit lui-même comme « un langage formulaire de la pensée pure ». On ne soulignera jamais assez que l'intention profonde de Frege n'est pas de créer une langue artificielle de plus, destinée à remplacer l'allemand et d'autres langues, mais de proposer une idéographie ou écriture conceptuelle susceptible d'être le moyen d'expression le plus fidèle possible de la 'pensée pure'. Cela est vrai de toutes les 'langues' que proposent les logiciens, de Leibniz à Frege : cette langue logique est uniquement une écriture, une notation écrite du raisonnement; elle n'a absolument pas les propriétés d'une langue orale. La meilleure preuve en est que ce 'langage formulaire' peut se lire en n'importe quelle langue naturelle ${ }^{12}$.

Pour lever toute ambiguïté sur l'objectif de Frege, nous indiquons ci-dessous quelques uns des signes et des notions que Frege a introduits et qui ont fait école :

1) $\vdash$ est le premier signe qu'il introduit et qu'il place devant une proposition : la barre horizontale représente le signe de contenu et la barre verticale le signe d'assertion ;

, est le même signe double, comportant une négation, qui se lit: « ce n'est pas le cas »

$\equiv$ est le signe d'identité de contenu entre deux expressions.

En combinant ces différents signes simples, Frege obtient d'autres notions logiques (voir Kneale : 482).

2) Frege rejette le dogme 'sujet - prédicat' qui règne sur la logique depuis Aristote. Certes, il peut y avoir une différence rhétorique ${ }^{13}$ entre

Les Perses furent défaits par les Grecs,

Les Grecs défirent les Perses

mais le contenu conceptuel reste le même, ce qui a pour conséquence que les termes de 'sujet' et d'objet' sont sans incidence sur le prédicat. Frege remplace alors les deux termes de sujet et de prédicat, hérités de la tradition grammaticale, par deux notions nouvelles, empruntées aux mathématiques: celle de fonction et celle d'argument de la fonction, telles que $\mathrm{y}=\mathrm{f}(\mathrm{x})$, dont il démontre l'avantage incontestable à partir des énoncés quantifiés ${ }^{14}$. 


\subsection{Les idées de Frege dans la Begriffsschrift et la langue universelle}

Le livre de Frege passa inaperçu, comme d'ailleurs bon nombre d'autres ouvrages que Frege publia par la suite. La Begriffsschrift n'a pas été relayée comme elle aurait dû l'être : aucun spécialiste allemand n'a manifesté un intérêt particulier pour l'ouvrage, bien au contraire; et en France, mis à part un bref compte-rendu de Paul Tannery (1843-1904) dans la Revue de Métaphysique et de Morale, mais sans en saisir la portée réelle, paraphrasant le titre en "représentation écrite des concepts " ${ }^{15}$, et un échange épistolaire sans intérêt avec Eugène Ballue, Frege n'a jamais eu le soutien qu'il attendait en France. Notamment Louis Couturat (1868-1914) n'a jamais tenu les promesses qu'il avait faites à Frege de faire des recensions de ses ouvrages et de diffuser ses idées.

Une question reste même posée : Pour quelles raisons Couturat, le logicien professeur d'université, en est-il venu à abandonner la voie tracée par la Begriffsschrift et la collaboration souhaitée avec Frege pour finalement soutenir une langue auxiliaire comme l'ido, issu de l'espéranto ? Une réponse possible serait qu'en 1901 il soutient sa thèse sur La logique de Leibniz et que Leibniz, dans ses recherches sur la langue universelle, ne perd jamais de vue les langues vernaculaires, qu'il tentera de les simplifier, notamment le latin, pour aboutir à une langue uniforme, destinée à un apprentissage plus facile.

Toujours est-il que la seule possibilité de collaboration qui s'annonçait pour Frege a été délibérément détournée de objectif initial par Couturat, qui - il faut le dire - n'était sans doute pas à la hauteur des vues originales et novatrices de Frege.

\subsection{Quelques critiques de la Begriffsschrift}

La position de Ludwig Wittgenstein (1889-1951) dans le Tractatus (1921) est importante car elle révèle indirectement des lacunes et des limites inhérentes à l'Idéographie de Frege, comme à toute idéographie : «Les propositions peuvent représenter l'ensemble de la réalité, mais elles ne peuvent représenter que ce qu'elles doivent avoir de commun avec la réalité pour pouvoir la représenter - la forme logique. » (Tractatus 4.12)

Et Récanati enchaîne : «Ce qui échappe ainsi au langage, ce qui n'est pas représentable, ce qui ne peut être dit, cela, affirme Wittgenstein, peut seulement être montré. Le langage est universel, parce qu'il n'y a jamais qu'un langage (le langage) par delà la diversité des langues naturelles qui n'en sont jamais, comme la Begriffsschrift qui se distingue d'en révéler au plus près l'essence, que des incarnations; mais le langage n'est pas universel, parce qu'il ne permet pas de tout dire.» (Critique : 780) Certes Russell essaiera de sauvegarder le caractère dicible du langage en faisant appel à la multiplication des langages; mais on peut se demander si cette multiplication des langages n'est pas un artifice consistant finalement à repousser indéfiniment le problème du dicible. 


\section{Un sytème mixte : le latino sine flexione de peano}

Giuseppe Peano (1858-1936), mathématicien et logicien italien, avait posé des prémisses excellents, se situant à mi-chemin entre les langues naturelles et la logique, c'est-à-dire prenant appui d'un côté sur les langues naturelles et planifiant leur fonctionnement selon des principes logiques; mais, en fin de compte, les résultats obtenus sont relativement décevants, notamment au plan grammatical et syntaxique.

\subsection{L'intérêt du projet de Peano}

Peano avait pour objectif essentiel de réduire le fossé qui se creusait inévitablement entre comparatisme et langue artificielle; dans cette entreprise, il avait quand même un point d'ancrage solide, car l'indo-européen, le fleuron du comparatisme, est pour une large part une langue artificielle, car reconstruite. Les racines indo-européennes pourront servir de base à une langue internationale moderne : d'où l'importance de la dérivation chez Peano.

En fait, Peano cherche à concilier deux objectifs difficilement compatibles

- prenant comme référence en 1903 L'introduction à l'étude comparative des langues indoeuropéennes d'Antoine Meillet, il cherche à donner une base de linguistique comparée à sa langue construite, ce qui lui évite de faire appel à l'anglais, comme le feront plus tard Couturat et Jespersen ;

- mais dans le même temps, il souhaite que cette langue soit obtenue par «application de la seule logique mathématique ».

"Le latin sans flexion" est une langue artificielle obtenue par des modifications brutales et drastiques: suppression des flexions, remplacement des cas par des prépositions, suppression du genre et du nombre, suppression des marques de personnes, temps, modes, etc. Ce faisant, on pourrait croire que Peano réinvente les langues d'Asie du sud-est; mais il ne fait qu'anticiper en accentuant le trait sur l'évolution vers le type analytique qui est déjà à l'œuvre dans les langues occidentales.

\subsection{Le projet de Peano à son époque}

On constate, avec une petite déception, que ce projet présente moins d'originalité qu'on serait tenté de croire au premier abord. Sans avoir procédé à un recensement complet des ouvrages sur la question, nous avons connaissance d'une publication de même date que celle de Peano - dont il aurait d'ailleurs pu s'inspirer (?). Il s'agit de l'ouvrage de Ch. André ${ }^{16}:$ Le latin et le problème de la langue internationale (Paris, Le Soudier, 1903). Ce qui nous intéresse ici, c'est la simplification radicale qu'André fait subir au latin.

Il propose de remédier à la fois à la pauvreté du vocabulaire et à la complexité de la grammaire en enrichissant l'un et en simplifiant l'autre : pour le lexique, il propose la création de termes à désinence latine, donc obtenus par dérivation et la latinisation de néologismes grecs; pour la morphologie, la réduction est importante et touche les déclinaisons et la conjugaison; enfin, il rejette la syntaxe, de sorte que la phrase latine correspondrait au mouvement analytique de la phrase moderne (sujet - verbe complément direct, puis indirect). 

Otto Jespersen (1860-1943), spécialiste initialement de langues romanes, puis d'anglais pour des raisons de carrière, auteur d'une œuvre considérable, dont les ouvrages les plus importants restent toujours d'actualité : Phonetische Grundfragen (1904), How to Teach a Foreign language (1904), Growth and Structure of the English Language (1905), Modern English Grammar en 7 volumes (1909-1949), Sprogets Logik (1913), The Philosophy of Grammar (1924), Analytic Syntax (1937).

Or Jespersen s'est engagé très tôt dans la création et la diffusion des langues auxiliaires internationales, devenant membre actif de la Délégation et collaborant avec Edward Sapir (1844-1939) aux recherches sur l'Ido, avant de fabriquer lui-même une langue artificielle, le Novial en 1928. En 1908, rédigeant la Préface, écrite en français, à un 
Dictionnaire International-Français (de L. de Beaufront et L. Couturat), il expose avec grande clarté ses motifs en faveur d'une langue internationle et les avantages qu'elle doit offrir.

Jespersen commence par énoncer les deux raisons essentielles, selon lui, l'une politique, l'autre linguistique, pour lesquelles il est exclu « de choisir une des langues naturelles comme langue internationale pour tout le monde »:

- «La première raison est que par là on favoriserait indûment un peuple aux dépens de tous les autres. On violerait ainsi le principe d'impartialité qui doit régner dans tous les rapports internationaux. $»^{19}$

- «La seconde raison, c'est que n'importe quelle langue est trop difficile pour tous les étrangers. Toutes les langues existantes fourmillent de difficultés de prononciation, d'orthographe, de grammaire, de lexique et surtout de phraséologie. ${ }^{20}$

Puis, il en arrive à la formule recherchée pour définir les propriétés que doit posséder la langue internationale ; cette formule paraît être d'une simplicité inattendue :

la meilleure langue internationale est celle qui présente la plus grande facilité pour le plus grand nombre d'hommes.

mais Jespersen la justifie aussitôt par des considérations d'ordre scientifique, placées dans une note: "C'est en vérité le même principe qui m'a servi de base dans deux travaux sur les langues naturelles; voir Progress in Language, London, 1894, et Phonetische Grundfragen, Leipzig, 1904. »

Et, dans les autres parties de sa Préface, il entreprend de démontrer de quelle manière peut s'exercer cette simplicité et cette facilité, obtenues par un examen attentif des langues naturelles et que l'on peut résumer en quelques points essentiels :

- «la règle de la plus grande facilité entraîne nécessairement le principe de l'orthographe phonétique ", qui réduit l'arbitraire du signifiant ;

- pour ce qui est du vocabulaire, le principe à respecter est celui «du maximum d'internationalité dans le choix de la majorité des racines ", qu'il est possible d'évaluer.

- en ce qui concerne la grammaire, c'est « la parfaite régularité » qui doit régner ; et Jespersen d'observer : « il serait certainement à désirer que les désinences de la grammaire pussent être aussi internationales que la plupart des radicaux, mais nos langues sont à cet égard si différentes l'une de l'autre qu'on arriverait au chaos si on voulait se guider par une statistique analogue à celle qui nous a servi pour le vocabulaire. »

Il est certain que Jespersen reste assez vague sur la morphologie : doit-elle être abolie ou peut-elle subsister en partie? Il paraît opter pour la seconde solution. Et surtout, lui qui va devenir un remarquable spécialiste de syntaxe, il reste muet sur les questions de construction et d'ordre des éléments.

\subsection{L'hostilité des néo-grammairiens aux projets de langue internationale}

Deux néo-grammairiens parmi les plus connus et les plus représentatifs, Karl Brugmann (1849-1919) et August Leskien (1840-1916), manifestent une hostilité ouverte en publiant, à la demande de la 'Königliche Sächsische Gesellschaft der Wissenschaften' à Leipzig, une brochure intitulée Zur Kritik der künstlichen Weltsprachen (Strassburg, Trübner, 1907), qui contient les principales critiques adressées aux langues internationales. 
59 Au début de son article, Brugmann revient sur le contexte général et les conditions dans lesquelles se sont développées les langues artificielles, notamment le volapük, qui s'est littéralement effondré après avoir connu un essor ultra-rapide entre 1879 et 1889 , mobilisant deux millions et demi d'adeptes. Mais les critiques exprimées à la fois par Brugmann, par Leskien et par Gustav Meyer se concentrent d'abord sur la question la plus aisée à épingler: la facilité plus ou moins grande d'apprentissage des langues artificielles, que ce soit le volapük ou l'espéranto, qui est mise en cause par les deux néo-grammairiens dans plusieurs domaines. En phonétique, ils reprochent à des langues artificielles destinées à servir à la communication orale de présenter un grand nombre de sons difficiles à articuler par des Occidentaux et de vouloir accentuer les mots sur la syllabe pénultième, ce qui aura inévitablement pour conséquence d'affaiblir le timbre des voyelles finales. Le vocabulaire et la morphologie de l'espéranto doivent beaucoup aux langues romanes. Au plan syntaxique, le problème essentiel, formulé en termes actuels, auquel n'échappe d'ailleurs pas la typologie des langues, concerne la non-isomorphie des catégories entre les langues naturelles : quelle doit alors être le choix de langue internationale? Leskien regrette le manque d'expérience des langues chez Zamenhof.

La seconde critique importante affiche une concordance assez inattendue, mais pas surprenante au fond, entre les néo-grammairiens Brugmann et Paul Diels d'une part et Saussure d'autre part. Les deux néo-grammairiens doutent qu'une langue artificielle puisse réellement concurrencer une langue naturelle. En effet, Saussure nous explique que « celui qui en crée une [= langue artificielle] la tient en main tant qu'elle n'est pas en circulation ; mais dès l'instant qu'elle remplit sa mission et devient la chose de tout le monde, le contrôle échappe. L'espéranto est un essai de ce genre; s'il réussit, échappera-t-il à la loi fatale? Passé le premier moment, la langue entrera très probablement dans sa vie sémiologique; elle se transmettra par des lois qui n'ont rien de commun avec celles de la création réfléchie, et l'on ne pourra plus revenir en arrière. L'homme qui prétendrait composer une langue immuable, que la postérité devrait accepter telle quelle, ressemblerait à la poule qui a couvé un œuf de canard : la langue créée par lui serait emportée bon gré mal gré par le courant qui entraîne toutes les langues.» (CLG : 111) Et Brugmann d'ajouter : "La langue est, comme tout acte culturel, soumise à l'évolution et au changement continuels. Dans toute sorte de langues, quand elle est répandue sur un terrain ou sur une communauté de quelque dimension qu'ils soient, entrent constamment en action aussi bien des forces centrifuges, différenciatives que des forces centripètes, nivelantes. [...] Les résultats de l'action de ces deux tendances diamétralement opposées représentent pour chaque type de langue, que nous avons l'habitude de considérer comme une unité, la partie principale de leur histoire. [...] Dans ces conditions, l'important pour nous est que la langue étrangère ${ }^{21}$ ne reste jamais pure, ni au plan phonétique, ni au plan syntaxique, ni au plan phraséologique 22 .» (1907: 23-24).

61 La position adoptée par Fr. Nietzsche (1844-1900), lorqu'il compare implicitement la grammaire historique avec la langue internationale ${ }^{23}$, résume assez bien la situation à l'aube du xx siècle: "Wozu hätte auch die Sprachwissenschaft ein Jahrhundert lang die Gesetze der Sprachen studiert und das Notwendige, Wertvolle, Gelungene an jeder einzelnen Sprache abgeschätzt ?» (Menschliches, Allzumenschliches, 1878). 

que les prétentions des auteurs ont chuté à chaque siècle : si au XVII ${ }^{\mathrm{e}}$ et au XVII ${ }^{\mathrm{e}}$ siècles, on évoque sans complexe une "langue universelle ", vieux rêve de l'humanité, le Xix ${ }^{e}$ siècle se contente de parler de "langue internationale » à connotation politique et le $\mathrm{xx}^{\mathrm{e}}$ siècle est encore plus en retrait avec la dénomination de «langue auxiliaire », qui évoque un simple outil pour les besoins de la communication scientifique, commerciale, etc. En fait la langue artificielle - qui est une dénomination finalement assez neutre - se heurte aux langues naturelles, qui ont l'immense mérite d'exister et d'occuper le terrain. La question de soutenir ou de combattre la langue artificielle a divisé la communauté des linguistes : dans le camp des adeptes, on rencontre Hugo Schuchardt (1842-1927), Professeur à Graz, Max Müller (1823-1900), Professeur à Oxford, Jan Baudouin de Courtenay (1845-1929), Professeur à Saint-Petersbourg et à Varsovie, Otto Jespersen (1860-194), Professeur à Copenhague, etc. ; le camp des adversaires compte la plupart des néo-grammairiens ou des proches, entre autres Karl Brugmann (1848-1919), Professeur à Leipzig, August Leskien (1840-1916), Professeur à Leipzig, Gustav Meyer (1850-1900), Hermann Diels (1848-1922). Mais cette position porte uniquement sur les langues artificielles fabriquées à partir des langues naturelles, vivantes ou mortes, car les linguistes de cette époque ignorent tout ou à peu près du travail des logiciens.

63

Lopposition fondamentale entre langue naturelle et langue artificielle ne met pas seulement en cause des personnes; c'est un vrai débat d'idées, dont les racines plongent dans l'esprit et le cœur des hommes. Le premier critère qui les distingue est bien le temps comme dimension historique et véhicule de la mémoire. En effet, une langue naturelle est ce que l'on appelle en allemand «eine historische gewordene Sprache »: c'est une langue qui est chargée d'évolution et d'histoire et qui s'inscrit dans la mémoire collective du peuple ou de la communauté qui en est le récipiendiaire, alors qu'une langue artificielle est au sens fort du mot une langue 'étrangère', venue de l'extérieur, qui n'a aucune implantation stable, aucune connivence avec la société et ne peut donc briguer ce statut d' "institution sociale", auquel Saussure attache tant d'importance. Le second critère est beaucoup plus difficile à évaluer : comment juger du progrès d'une langue? L'évolution naturelle des langues peut-elle être considérée comme un progrès, et de quel point de vue ? Une langue artificielle représente-t-elle un progrès par rapport à une langue naturelle? Autant de questions auxquelles la linguistique est incapable de répondre sur des critères sérieux et objectifs, car un tel jugement reposerait naturellement sur une idéologie. Jespersen (1894) avait pensé avoir montré que l'évolution des langues naturelles, en l'occurrence l'anglais, marquait un progrès allant vers une simplicité plus grande; cet avis soulèverait une longue discussion. Les tenants des langues artificielles veulent reconnaître un progrès dans l'introduction d'un telle langue, parce qu'elle faciliterait la communication entre les hommes de langues différentes, ce qui n'est qu'un truisme.

Quels que puissent être les sentiments du linguiste, l'échec des projets de langue internationale est bien consommé, et pour longtemps. Nous en subissons aujourd'hui encore, à presque un siècle de distance, les conséquences, car une langue est à nouveau considérée comme un produit d'exportation et un gage d'expansion. Au conflit entre 
les langues artificielles, a fait place tout simplement la guerre des langues ${ }^{24}$ - qu'elles voulaient précisément éviter.

\section{BIBLIOGRAPHIE}

de Beaufront, L. \& Couturat, L. (1908) : Dictionnaire international-français, avec une Préface d'Otto Jespersen, Paris, Delagrave.

Brugmann, Karl \& Osthoff, Hermann (1878-1890) : Morphologische Untersuchungen auf dem Gebiete der indogermanischen Sprachen, 5 vol., Leipzig, Hirzel.

Brugmann, Karl \& Leskien, August (1907) : Zur Kritik der künstlichen Weltsprachen. Strassburg, Trübner.

Cortès, C. \& Rousseau A. éd. (1999) : Catégories et connexions, Lille, Septentrion.

Couturat, Louis (1901) : La Logique de Leibniz d'après des documents inédits, Paris, Alcan.

Couturat, Louis \& Léau, Léopold (1903) : Histoire de la langue universelle, Paris, Hachette.

Couturat, L., Jespersen, O., Lorenz, R. Ostwald, W., Pfaundler, L. (1909) : La Langue internationale et la science (trad. fr.), Paris, Delagrave.

Eco, Umberto (1994) : La Recherche de la langue parfaite dans la culture européenne, Paris, Le Seuil.

Frege, Gottlob (1879) : Begriffsschrift, eine der arithmetischen nachgebildete Formelsprache des reinen Denkens, Halle, Louis Nebert.

Frege, Gottlob (1999) : Idéographie (trad. fr.), Paris, Vrin.

Jespersen, Otto (1894) : Progress in Language with Speciel Reference to English, London, Sommendheim.

Kneale, William \& Martha (1962) : The Development of Logic, Oxford, Clarendon Press.

Leibniz, G.W. (1679-1697) : L'Harmonie des langues (édition bilingue), Paris, Le Seuil.

Le mythe de la langue universelle, in : Critique 387-388, août-septembre 1979.

Rossi, Paolo (1983) : Clavis Universalis. Arts de la mémoire, logique combinatoire et langue universelle de Lulle à Leibniz, trad. fr. 1993, Grenoble, Million.

Rousseau, André (1994) : « Accueil, devenir et actualité de la pensée logique de Gottlob Frege. Contribution à la sémantique logique », in : J. Quillien (éd.), La Réception de la philosophie allemande en France aux XIX ${ }^{e}$ et XX $X^{e}$ siècles, Lille, P.U.L., p. 135-170.

Rousseau, André (1999a) : « La sémantique logique de Gottlob Frege » in : H.S. Gill \& G. Manetti (éd.) Signs and Signification, vol. I. New Delhi, Bahri Publications. p. 15-26.

Rousseau, André (1999b) : « Syntaxe et typologie des langues: quelques jalons », in : C. Cortès \& A. Rousseau (éd.), Catégories et connexions, Lille, Septentrion, p. 237-245.

Savatovsky, Dan (1989) : « Les linguistes et la langue internationale : 1880-1920», in : H.E.L. 11, 2, p. 37-65. 
Savatovsky, Dan (1992) : «Peano lecteur de Meillet. Logique, grammaire comparée et langue internationale » in : Langages 107 p. 96-111.

\section{NOTES}

1. Il faudrait naturellement évoquer également Condillac (1714-1780), qui a laissé une œuvre posthume, intitulée La langue des calculs (1797), dans laquelle « des observations, faites sur les commencemens et les progrès de cette langue, démontrent les vices des langues vulgaires, et font voir comment on pourrait, dans toutes les sciences, réduire l'art de raisonner à une langue bien faite ".

2. L'existence des langues artificielles présuppose celle des langues naturelles dont il sera également question, ne serait-ce que par opposition.

3. Commpe nous l'expliquons par la suite, 'mixte' signifie pour nous que le projet s'appuie à la fois sur les langues naturelles et sur la logique.

4. À la fin du XIX ${ }^{\mathrm{e}}$ siècle, les thèses présentées à la Sorbonne étaient en général encore écrites en latin.

5. Il faut aussi rappeler que l'adjectif «parfait» est précisement la dénomination qui désigne le sanskrit.

6. Consulter à ce sujet l'article d'Alain Pons «Les langues imaginaires dans les utopies de l'âge classique » Critique 387-388 : 720-735.

7. Mais traduit en allemand par Lommel seulement en 1931.

8. Citons, à titre anecdotique, le Lingwo internaciona (1908) de René de Saussure (1868-1943), un frère cadet de Ferdinand de Saussure.

9. Couturat et Léau en citent une bonne partie dans leur Histoire de la langue universelle, mais seulement jusqu'à 1903 ou 1909, suivant les éditions.

10. Raoul Guérin de la Grasserie (1839-1914), juriste et linguiste aux multiples publications, avait publié dix ans plus tôt, en 1897 : De la possibilité et des conditions de la langue internationale. Paris, E. Leroux.

11. Frege exprime parfois une position plus nuancée.

12. Cet ouvrage vient d'ailleurs d'être enfin traduit en français par Corinne Besson: Idéographie, Paris, Vrin. 1999.

13. Nous présentons ici l'analyse de Frege, sans nous impliquer dans cette démarche.

14. De toutes les nouveautés introduites par Frege dans sa Begriffsschrift, celle concernant les quantifieurs ou quantificateurs est de loin la plus importante.

15. Au milieu de son compte-rendu, Tannery a placé une phrase qui en dit long sur le jugement qu'il porte sur la Begriffsschrift : « les explications sont insuffisantes, les notations d'une excessive complexité et les applications ... restent à l'état de promesses. ", enchaînant aussitôt : «Le Dr. Frege ne se fait d'ailleurs guère d'illusion sur l'accueil que recevra probablement son présent travail. "

16. Il s'agit du sous-bibliothécaire à l'Université de Lyon.

17. Ce recenseur pour la Revue de Philologie française (17, 1903. pp. 105-113) n'est autre que François Vézinet, le gendre de Léon Clédat.

18. Rappelons au passage que 1879 est aussi l'année où Frege publia sa Begriffsschrift.

19. On souhaiterait qu'aujourd'hui les responsables politiques fassent preuve de la même lucidité et que certains pays dominateurs s'inspirent de ces principes dictés par le bon sens.

20. Nous avons tenu à citer fidèlement le texte de Jespersen, qui reste actuel.

21. 'Fremdsprache' désigne ici chez Brugmann la langue artificielle introduite dans une communauté linguistique. 
22. Nous avons traduit en français ce passage de Brugmann.

23. Il ne faut pas oublier que Nietsche a été Professeur de philologie des langues classiques à Bâle. 24. Et j'aimerais ajouter : 'dans un monde qui ne s'embarrasse pas des scrupules qui honoraient nos aïeux.'

\section{RÉSUMÉS}

Les langues naturelles sont par nature inscrites dans le temps, ce qui les soumet ipso facto à la double problématique de la mémoire et du progrès. Les langues artificielles - ou du moins les projets que nous connaissons -, qui ont proliféré de 1880 à 1930, sont d'origine diverse : soit purement logique, comme la Begriffsschrift (1879) de Frege, soit mixte, comme le Latino sine flexione (1903) de Peano, soit sur base de langue naturelle comme le Volapük, l'Esperanto ou l'Ido. Ces langues, qui prétendaient signifier un progrès par rapport aux langues naturelles, ont délibérément négligé la mémoire de la communauté linguistique dans laquelle elles souhaitaient s'implanter, ce qui représente certainement la cause profonde de leur échec.

Die natürlichen Sprachen unterliegen von Natur aus der Zeit, weshalb sie ipso facto der doppelten Problematik des Gedächtnisses und des Fortschritts unterworfen sind. Die künstlichen Sprachen - zumindest die Projekte die wir kennen - von denen zwischen 1880 und 1930 schon eine Vielzahl in Erscheinung traten, haben verschiedene Grundlagen: Entweder beruhen sie auf logischen Überlegungen, wie die Begriffsschrift (179) von Frege, oder sie sind gemischten Ursprungs, wie Latino sine flexione (1903) von Peano, oder sie beruhen auf der Basis einer natürlichen Sprache, wie Volapük, Esperanto oder Ido. Diese Sprachen, die gegenüber den natürlichen Sprachen einen Fortschritt darstellen wollten, haben bewußt das Gedächtnis der Sprachgemeinschaft nicht berücksichtigt, in die sie sich integrieren wollten, was sicherlich den Grund ihres Mißerfolges bedeutet.

\section{AUTEUR}

\section{ANDRÉ ROUSSEAU}

Université Charles-de-Gaulle Lille 3 